\title{
Realcompactness and spaces of vector-valued functions
}

\author{
by \\ Jesús Araujo (Santander)
}

\begin{abstract}
It is shown that the existence of a biseparating map between a large class of spaces of vector-valued continuous functions $A(X, E)$ and $A(Y, F)$ implies that some compactifications of $X$ and $Y$ are homeomorphic. In some cases, conditions are given to warrant the existence of a homeomorphism between the realcompactifications of $X$ and $Y$; in particular we find remarkable differences with respect to the scalar context: namely, if $E$ and $F$ are infinite-dimensional and $T: C^{*}(X, E) \rightarrow C^{*}(Y, F)$ is a biseparating map, then the realcompactifications of $X$ and $Y$ are homeomorphic.
\end{abstract}

1. Introduction. Let $\mathbb{K}=\mathbb{R}$ or $\mathbb{C}$. Given a completely regular space $X$, and a $\mathbb{K}$-normed space $E, C(X, E)$ and $C^{*}(X, E)$ denote the spaces of continuous functions and bounded continuous functions on $X$ taking values in $E$, respectively. $C(X)$ and $C^{*}(X)$ are the spaces $C(X, \mathbb{K})$ and $C^{*}(X, \mathbb{K})$, respectively.

Sometimes an algebraic relation between spaces of continuous functions $C(X)$ and $C(Y)$ may determine some kind of topological link between the spaces $X$ and $Y$. For instance, it is well known that the existence of a ring isomorphism between the spaces $C(X)$ and $C(Y)$ produces a homeomorphism between the realcompactifications of $X$ and $Y$ (see [5, pp. 115-118] and [6]). Some kind of weakening of the conditions does not alter the result: if we replace "ring isomorphism" by "biseparating map" (see definition below), we keep the conclusion on the existence of a homeomorphism between the realcompactifications ([1]). On the other hand, it is also true that if the realcompactifications $v X$ and $v Y$ are homeomorphic through a homeomorphism $h: v Y \rightarrow v X$, then there exists a biseparating map (in fact, a ring isomorphism) between $C(X)$ and $C(Y)$, as the map sending each $f \in C(v X)$ to $f \circ h$ $\in C(v Y)$ can be identified with an isomorphism between $C(X)$ and $C(Y)$.

2000 Mathematics Subject Classification: Primary 54C35; Secondary 54C40, 54D60, $46 \mathrm{E} 40$.

Research partially supported by the Spanish Dirección General de Investigación Científica y Técnica (DGICYT, PB98-1102). 
Of course, if we study ring isomorphisms between $C^{*}(X)$ and $C^{*}(Y)$, the conclusions we obtain are in general poorer, in the sense that we can no longer conclude the existence of a homeomorphism between the realcompactifications of $X$ and $Y$, but just that the Stone-Cech compactifications of $X$ and $Y$ are homeomorphic. And this because every function $f \in C^{*}(X)$ can be extended to a continuous function in $C(\beta X)$. This implies that every ring isomorphism between $C^{*}(X)$ and $C^{*}(Y)$ can be regarded as one between $C(\beta X)$ and $C(\beta Y)$. Similar arguments also apply to biseparating maps between spaces of bounded continuous functions: the characteristics of these spaces yield a poor link between $X$ and $Y$. As an example, if we take a realcompact space $X$ which is not compact and define $Y:=\beta X$, then the map $T: C^{*}(X) \rightarrow C^{*}(Y)$ sending each $f \in C^{*}(X)$ to its extension over $\beta X$ is clearly a biseparating map. Nevertheless $X$ and $\beta X$ are not homeomorphic. This example also illustrates the fact that, in general, the existence of a biseparating map from $C^{*}(X)$ onto $C^{*}(Y)$ does not imply the existence of a similar map between $C(X)$ and $C(Y)$. Indeed, if such a map existed, we would conclude in our example that $X$ and $\beta X$, which are both realcompact, should be homeomorphic, contrary to our assumptions.

And what about spaces of vector-valued functions? Of course, in this context ring isomorphism does not make sense, but a close concept of biseparating map can be introduced.

In particular, a natural question arises: what happens if we consider biseparating maps $T: C^{*}(X, E) \rightarrow C^{*}(Y, F)$ ? Clearly if $E$ and $F$ are infinitedimensional, we can no longer act as in the scalar-valued case, because since $E$ and $F$ are not locally compact, bounded continuous functions cannot be extended to functions on $\beta X$ or $\beta Y$ attaining values in $E$ or $F$. On the other hand, classical techniques involving the study of ideals are no longer useful because our spaces do not have a ring structure.

What we obtain in this paper is a different conclusion. In our new context we are in a position to prove that the existence of a biseparating map between $C^{*}(X, E)$ and $C^{*}(Y, F)$ implies that not only the Stone-Cech compactifications of $X$ and $Y$ are homeomorphic, but so also are their realcompactifications, a result that, as we mentioned above, is far from being true in the scalar case. On the other hand, this result is the best we can expect: that is, we cannot expect a homeomorphism between $X$ and $Y$.

Of course, we do not restrict our study to these special spaces, but we also obtain similar results for a large class of spaces, many of them containing unbounded functions. For instance, if we deal with spaces which do not contain "many" functions, the conclusions we get apply not to the spaces $X$ and $Y$, but to some compactifications of them. But even in these cases, the structure of $X$ and $Y$ provides sometimes a strong link between them. In 
particular, this happens when we study biseparating maps between spaces of uniformly continuous bounded functions.

2. Definitions and notation. Throughout the paper $X$ and $Y$ will be completely regular topological spaces, and $E$ and $F$ will be $\mathbb{K}$-normed spaces. If $X$ is also a complete metric space, we introduce $C_{\mathrm{u}}^{*}(X, E)$ as the space of uniformly continuous bounded functions defined on $X$ and taking values in $E$. Also in this case $C_{\mathrm{u}}^{*}(X)=C_{\mathrm{u}}^{*}(X, \mathbb{K})$.

Definition 2.1. Given $f \in C(X, E)$, we define the cozero set of $f$ as

$$
c(f):=\{x \in X: f(x) \neq 0\} .
$$

For the following two definitions, we assume that $A, B$ are subrings of $C(X)$ and $C(Y)$, respectively, and that $A(X, E) \subset C(X, E), A(Y, F) \subset$ $C(Y, F)$ are an $A$-module and a $B$-module, respectively.

Definition 2.2. A map $T: A(X, E) \rightarrow A(Y, F)$ is said to be separating if it is additive and $c(T f) \cap c(T g)=\emptyset$ whenever $f, g \in A(X, E)$ satisfy $c(f) \cap c(g)=\emptyset$. Moreover $T$ is said to be biseparating if it is bijective and both $T$ and $T^{-1}$ are separating.

Equivalently, an additive map $T: A(X, E) \rightarrow A(Y, F)$ is separating if $\|(T f)(y)\| \cdot\|(T g)(y)\|=0$ for all $y \in Y$ whenever $f, g \in A(X, E)$ satisfy $\|f(x)\| \cdot\|g(x)\|=0$ for all $x \in X$. Notice that in particular every ring isomorphism between $C(X)$ and $C(Y)$ is clearly a biseparating map. Linearity of maps will be assumed at no point of this paper.

Definition 2.3. Let $T: A(X, E) \rightarrow A(Y, F)$ be a map and suppose that $\gamma X$ is a compactification of $X$. A point $x \in \gamma X$ is said to be a support point of $y \in Y$ if, for every neighborhood $U$ of $x$ in $\gamma X$, there exists $f \in A(X, E)$ satisfying $c(f) \subset U$ such that $(T f)(y) \neq 0$.

For a continuous map $f: X \rightarrow \mathbb{K}, f^{\beta X}: \beta X \rightarrow \mathbb{K} \cup\{\infty\}$ stands for the continuous extension to $\beta X$ (the Stone-Cech compactification of $X$ ), mapping into $\mathbb{K} \cup\{\infty\}$. In particular, given a continuous map $f: X \rightarrow E$, $\|f\|^{\beta X}$ is the continuous extension to $\beta X$ of $\|\cdot\| \circ f: X \rightarrow \mathbb{K} \cup\{\infty\}$. In the same way, if $\gamma X$ is a compactification of $X$, and $f: X \rightarrow \mathbb{K}$ is a continuous function which can be continuously extended to a map from $\gamma X$ into $\mathbb{K} \cup\{\infty\}$, we will denote by $f^{\gamma X}$ this natural extension. Also, for $\mathbf{e} \in E$, $\widehat{\mathbf{e}}$ will stand for the function constantly equal to e. Finally, given $C \subset X$ and $D \subset \gamma X, \operatorname{cl} C$ and $\operatorname{cl}_{\gamma X} D$ will be their closures in $X$ and $\gamma X$, respectively.

Assume that $A$ is a subring of $C(X)$ which separates each point of $X$ from each point of $\beta X$. In $\beta X$, we introduce the equivalence relation $\sim$, defined as $x \sim y$ whenever $f^{\beta X}(x)=f^{\beta X}(y)$ for every $f \in A$. In this way, we obtain the quotient space $\gamma X:=\beta X / \sim$. It is easy to see that $\gamma X$ is a compactification of $X$, and that every $f \in A$ can be continuously extended 
to a map from $\gamma X$ into $\mathbb{K} \cup\{\infty\}$. Moreover, this extension is bounded if $f$ is bounded.

Suppose that $A(X, E) \subset C(X, E)$ is an $A$-module, where $A$ is a subring of $X$ which separates each point of $X$ from each point of $\beta X$. We say that $A(X, E)$ is compatible with $A$ if, for every $x \in X$, there exists $f \in A(X, E)$ with $f(x) \neq 0$, and if, given any points $x, y \in \beta X$ with $x \sim y$, we have $\|f\|^{\beta X}(x)=\|f\|^{\beta X}(y)$ for every $f \in A(X, E)$. It is clear that, in this case, for each $f \in A(X, E)$, there exists a continuous extension $\|f\|^{\gamma X}: \gamma X \rightarrow$ $\mathbb{K} \cup\{\infty\}$ of $\|\cdot\| \circ f$ to the whole space $\gamma X$.

A subring $A \subset C(X)$ is said to be strongly regular if given $x_{0} \in \gamma X$ and a nonempty closed subset $K$ of $\gamma X$ which does not contain $x_{0}$, there exists $f \in A$ such that $f^{\gamma X} \equiv 1$ on a neighborhood of $x_{0}$ and $f^{\gamma X}(K) \equiv 0$.

Examples. 1. Suppose that $X$ is a metric space. Then the spaces $C(X, E)$ and $C^{*}(X, E)$ are both $C^{*}(X)$-modules compatible with $C^{*}(X)$. Also, in this case, it is easy to see that $\gamma X=\beta X$.

2. Now suppose that $X$ is a complete metric space. It is easy to see that $C_{\mathrm{u}}^{*}(X, E)$ is a $C_{\mathrm{u}}^{*}(X)$-module compatible with $C_{\mathrm{u}}^{*}(X)$. In this case, in general, $\gamma X \neq \beta X$. On the other hand, it is immediate that we can embed isometrically our space $C_{\mathrm{u}}^{*}(X)$ in $C(\gamma X)$. Also $C_{\mathrm{u}}^{*}(X)$ is a closed subalgebra of $C(\gamma X)$ which separates points, contains constants and, when $\mathbb{K}=\mathbb{C}$, it is closed under complex conjugation. Then, by the Stone-Weierstrass theorem, it coincides with $C(\gamma X)$. Consequently, $C_{\mathrm{u}}^{*}(X)$ is a strongly regular ring.

3. Suppose that $\Omega$ is a (not necessarily bounded) open subset of $\mathbb{R}^{p}$ $(p \in \mathbb{N})$. For a Banach space $E$, consider the set $C^{n}(\Omega, E)$ of all $E$-valued functions whose partial derivatives up to order $n$ exist and are continuous $(n \geq 1)$. Take $A=C^{n}(\Omega, \mathbb{R})$, which is a strongly regular ring (see for instance [9, Corollary 1.2]). On the other hand, it is straightforward to see that $C^{n}(\Omega, E)$ is an $A$-module and that, in this case $\gamma \Omega=\beta \Omega$.

4. In a similar way, if $\Omega$ is a bounded open subset of $\mathbb{R}^{p}(p \in \mathbb{N})$ and $E$ is a Banach space, we define $C^{n}(\bar{\Omega}, E)$ as the subspace of $C^{n}(\Omega, E)$ of those functions whose partial derivatives up to order $n$ admit continuous extension to the boundary of $\Omega$. Clearly, we can view $C^{n}(\bar{\Omega}, E)$ as a subset of $C\left(\operatorname{cl}_{\mathbb{R}^{p}} \Omega, E\right)$. As above, $A=C^{n}(\bar{\Omega}, \mathbb{R})$ is a strongly regular ring for which $\gamma \Omega=\operatorname{cl}_{\mathbb{R}^{p}} \Omega$, and $C^{n}(\bar{\Omega}, E)$ is an $A$-module.

A subring $A \subset C(X)$ is said to be normal if given two disjoint closed subsets $K, L$ of $\beta X$, there exists $f \in A, 0 \leq f \leq 1$, satisfying $f^{\beta X}(K) \equiv 1$ and $f^{\beta X}(L) \equiv 0$. It is said to be local if $f \in C(X)$ belongs to $A$ whenever for every $x \in X$ there exist an open neighborhood $U(x)$ of $x$ and $f_{x} \in A$ such that $f \equiv f_{x}$ in $U(x)$.

It is clear that each normal subring $A$ of $C(X)$ is also strongly regular, and that every $A$-module $A(X, E) \subset C(X, E)$ must be compatible with $A$. 
In this paper we will assume we are in one of the following three situations.

Situation 1. $A(X, E) \subset C(X, E)$ and $A(Y, F) \subset C(Y, F)$ are an $A$ module and a $B$-module compatible with $A$ and $B$, respectively, where $A \subset$ $C(X)$ and $B \subset C(Y)$ are strongly regular rings. Also, in the case when $\gamma X \neq \beta X$ and $\gamma Y \neq \beta Y$, we assume that for every $x \in \beta X$ and $y \in \beta Y$, there exist $f \in A(X, E)$ and $g \in A(Y, F)$ satisfying $\|f\|^{\beta X}(x) \neq 0$ and $\|g\|^{\beta Y}(y) \neq 0$.

Situation 2. $A(X, E) \subset C(X, E)$ is an $A$-module and $A(Y, F) \subset$ $C(Y, F)$ is a $B$-module, where $A \subset C(X)$ and $B \subset C(Y)$ are normal local rings.

Situation 3. $E$ and $F$ are infinite-dimensional, and $A(X, E)=$ $C^{*}(X, E), A(Y, F)=C^{*}(Y, F)$.

Notice that when we are in Situations 2 or 3, then we are also in Situation 1. Also it is clear that when we are in Situations 2 or 3 , then $\gamma X=\beta X$ and $\gamma Y=\beta Y$.

We will denote by $v X$ the realcompactification of $X$.

Throughout the paper the word "homeomorphism" will be synonymous with "surjective homeomorphism".

3. Main results. We first state a general result. Even if we may not have many functions in our spaces we can link the structures of some compactifications of $X$ and $Y$.

ThEOREM 3.1. Suppose that we are in Situation 1. If $T: A(X, E) \rightarrow$ $A(Y, F)$ is a biseparating map, then $\gamma X$ and $\gamma Y$ are homeomorphic.

In some contexts, such as when $X$ and $Y$ have special structures, we can even ensure the existence of a homeomorphism between both spaces.

Corollary 3.2. Assume that we are in Situation 1. Suppose that $\gamma X=$ $\beta X$ and $\gamma Y=\beta Y$. If $X$ and $Y$ are first-countable spaces and $T: A(X, E) \rightarrow$ $A(Y, F)$ is biseparating, then $X$ and $Y$ are homeomorphic. In particular, if $X$ and $Y$ are open subsets of $\mathbb{R}^{p}$ and $\mathbb{R}^{q}$, respectively, then $p=q$.

Corollary 3.3. Let $A(X, E)=C_{\mathrm{u}}^{*}(X, E)$ and $A(Y, F)=C_{\mathrm{u}}^{*}(Y, F)$. If $T: A(X, E) \rightarrow A(Y, F)$ is a biseparating map, then $X$ and $Y$ are uniformly homeomorphic, that is, there exists a homeomorphism $h: Y \rightarrow X$ such that both $h$ and $h^{-1}$ are uniform maps.

Notice that Corollary 3.2 applies to Examples 1, 3 and 4. In a similar way, Corollary 3.3 applies to Example 2.

Finally we state the main result of the paper, which applies to a large family of spaces of vector-valued functions. 
Theorem 3.4. Suppose that we are in Situations 2 or 3 . If $T: A(X, E)$ $\rightarrow A(Y, F)$ is biseparating, then $v X$ and $v Y$ are homeomorphic.

In general we cannot conclude, in Theorem 3.4, that $X$ and $Y$ are homeomorphic, as the following example shows.

EXAMPLE. Take any space $X$ which is not realcompact (for instance $W\left(\omega_{1}\right):=\left\{\sigma: \sigma<\omega_{1}\right\}$, where $\omega_{1}$ denotes the first uncountable ordinal; see $[5,5.12])$, and a realcompact normed space $E$. Recall that every normed space (or, more generally, every metrizable space) of nonmeasurable cardinality is realcompact ([5, p. 232]), and the assumption that all cardinal numbers are nonmeasurable is consistent with the axioms of set theory ([3, p. 217]). Next, each continuous map $f: X \rightarrow E$ can be extended to a continuous map $f^{v X}: v X \rightarrow E$. Clearly the map sending each $f \in C(X, E)$ to $f^{v X} \in C(v X, E)$ is biseparating but $X$ and $v X$ are not homeomorphic.

4. Proofs I. In this section we assume that we are in Situation 1.

Lemma 4.1. For any $x \in X$, if $U$ is an open neighborhood of $x$ in $\gamma X$, then there exists $f \in A(X, E)$ such that $x \in c(f)$ and $c(f) \subset U$.

Proof. Take $g \in A(X, E)$ such that $x \in c(g)$, and $k \in A$ such that $k(x)=1$ and $k^{\gamma X} \equiv 0$ outside $U$. It is easy to see that $f:=g k \in A(X, E)$ does the job.

Lemma 4.2. Let $T: A(X, E) \rightarrow A(Y, F)$ be a biseparating map. For $f, g \in A(X, E)$, if $c(f) \subset c(g)$, then $c(T f) \subset \operatorname{cl}_{\gamma Y} c(T g)$.

Proof. Suppose that we can find $f, g \in A(X, E)$ with $c(f) \subset c(g)$ and $c(T f) \not \subset \mathrm{cl}_{\gamma Y} c(T g)$. Take $y \in c(T f), y \notin \mathrm{cl}_{\gamma Y} c(T g)$. By Lemma 4.1 there exists $k \in A(Y, F)$ such that $k(y) \neq 0$ and $c(k) \cap \operatorname{cl}_{\gamma Y} c(T g)=\emptyset$. Consequently, $c(k) \cap c(T g)=\emptyset$ and, since $T^{-1}$ is separating, we deduce that $c\left(T^{-1} k\right) \cap c(g)=\emptyset$. Using the fact that $T$ is separating we deduce that $c(k) \cap c(T f)=\emptyset$. Since this is a contradiction, we conclude that $c(T f) \subset$ $\operatorname{cl}_{\gamma Y} c(T g)$.

Lemma 4.3. Let $T: A(X, E) \rightarrow A(Y, F)$ be a biseparating map. Then for each $y \in Y$, there exists a unique support point of $y$ in $\gamma X$.

Proof. Take any $y \in Y$ and define $I_{y}:=\{f \in A(Y, F): y \in c(f)\}$. Now consider $H(y):=\bigcap_{f \in I_{y}} \mathrm{cl}_{\gamma X} c\left(T^{-1} f\right)$.

Claim 1. $H(y)$ is nonempty.

We are going to see that the family $\left\{\operatorname{cl}_{\gamma X} c\left(T^{-1} f\right): f \in I_{y}\right\}$ has the finite intersection property. Take $f_{1}, \ldots, f_{n} \in I_{y}$. By Lemma 4.1, we can consider $f \in I_{y}$ such that $c(f) \subset \bigcap_{i=1}^{n} c\left(\left\|f_{i}\right\|^{\gamma Y}\right)$. This gives us $c(f) \subset \bigcap_{i=1}^{n} c\left(f_{i}\right)$. Then, by Lemma 4.2 applied to $T^{-1}$, we see that $c\left(T^{-1} f\right) \subset \operatorname{cl}_{\gamma X} c\left(T^{-1} f_{i}\right)$ for every $i \in\{1, \ldots, n\}$, which implies that $\bigcap_{i=1}^{n} \operatorname{cl}_{\gamma X} c\left(T f_{i}\right) \neq \emptyset$. By the 
compactness of $\gamma X$, we conclude that $H(y)$ is nonempty, and the claim is proved.

Claim 2. For each $y_{0} \in Y, H\left(y_{0}\right)$ consists of just one point.

Assume on the contrary that there exist two different points $x_{1}, x_{2} \in$ $H\left(y_{0}\right)$. Take $f_{0} \in A(Y, F)$ such that $f_{0}\left(y_{0}\right) \neq 0$. By the definition of $H\left(y_{0}\right)$, both $x_{1}$ and $x_{2}$ belong to $\mathrm{cl}_{\gamma X} c\left(T^{-1} f_{0}\right)$. Consider a closed neighborhood $U_{2}$ of $x_{2}$ such that $x_{1} \notin U_{2}$. Since $A$ is strongly regular, we can take $g_{1} \in A$ such that $g_{1}^{\gamma X} \equiv 1$ on a neighborhood $U_{1}$ of $x_{1}$, and $g_{1}^{\gamma X} \equiv 0$ on $U_{2}$. It is clear that

$$
f_{0}=T\left(g_{1}\left(T^{-1} f_{0}\right)\right)+T\left(\left(1-g_{1}\right)\left(T^{-1} f_{0}\right)\right)
$$

and consequently $y_{0}$ belongs to the cozero set of one of the summands. We assume without loss of generality that $y_{0} \in c\left(T\left(g_{1}\left(T^{-1} f_{0}\right)\right)\right)$. Then, since $x_{2} \in H\left(y_{0}\right)$, it follows that $x_{2}$ belongs to $\operatorname{cl}_{\gamma X} c\left(g_{1}\left(T^{-1} f_{0}\right)\right)$, which is not true by construction.

We conclude that $H\left(y_{0}\right)$ contains just one point.

Claim 3. Given $y_{0} \in Y$, if $x \in H\left(y_{0}\right)$, then $x$ is a support point of $y_{0}$.

Consider an open neighborhood $U$ of $x \in H\left(y_{0}\right)$ in $\gamma X$. We have to prove that there exists $g_{0} \in A(X, E)$ such that $c\left(g_{0}\right) \subset U$ and $\left(T g_{0}\right)\left(y_{0}\right) \neq 0$.

Take $f_{0} \in A(Y, F)$ such that $f_{0}\left(y_{0}\right) \neq 0$. Of course, if $c\left(T^{-1} f_{0}\right)$ is contained in $U$, we get the result by defining $g_{0}:=T^{-1} f_{0}$, so we suppose this is not the case.

Take $g_{1} \in A$ such that $\operatorname{cl}_{\gamma X} c\left(g_{1}\right) \subset U$ and $g_{1}^{\gamma X} \equiv 1$ on a neighborhood of $x$. It is clear that

$$
T^{-1} f_{0}=g_{1} T^{-1} f_{0}+\left(1-g_{1}\right) T^{-1} f_{0},
$$

and so, as above,

$$
\left(T\left(g_{1} T^{-1} f_{0}\right)\right)\left(y_{0}\right) \neq 0 \quad \text { or } \quad\left(T\left(\left(1-g_{1}\right) T^{-1} f_{0}\right)\right)\left(y_{0}\right) \neq 0 .
$$

But notice that if the latter holds, since $x \in H\left(y_{0}\right)$ we should have $x \in$ $\mathrm{cl}_{\gamma X} c\left(\left(1-g_{1}\right) T^{-1} f_{0}\right)$, which is not the case. Consequently, defining $g_{0}:=$ $g_{1} T^{-1} f_{0}$ we are done.

The previous lemma allows us to define a map $h: Y \rightarrow \gamma X$ sending each point $y \in Y$ to its support point $h(y) \in \gamma X$.

Lemma 4.4. Let $T: A(X, E) \rightarrow A(Y, F)$ be a biseparating map. Suppose that $h(y)=x$ for some $y \in Y$, and that $f \in A(X, E)$ satisfies $\|f\|^{\gamma X} \equiv 0$ on a neighborhood of $x$. Then $T f \equiv 0$ on a neighborhood of $y$.

Proof. Take an open neighborhood $U$ of $x$ in $\gamma X$ such that $\|f\|^{\gamma X} \equiv 0$ in $U$. By the definition of support point, we can take $g \in A(X, E)$ such that $c(g) \subset U$ and $(T g)(y) \neq 0$. Since $T$ is biseparating, and $c(f) \cap c(g)=\emptyset$, we deduce that $T f \equiv 0$ in $c(T g)$, which is a neighborhood of $y$. 
Now the following corollary follows easily.

Corollary 4.5. Let $T: A(X, E) \rightarrow A(Y, F)$ be a biseparating map. Suppose that $y \in Y$, and that $f \in A(X, E)$ satisfies $(T f)(y) \neq 0$. Then $h(y) \in \operatorname{cl}_{\gamma X} c(f)$.

Lemma 4.6. Given a biseparating map $T: A(X, E) \rightarrow A(Y, F)$, the associated map $h: Y \rightarrow \gamma X$ is continuous, and its range is dense in $\gamma X$.

Proof. We shall see first that $h$ is continuous at every point of $Y$. Take $y_{0} \in Y$ and an open neighborhood $U$ of $h\left(y_{0}\right)$ in $\gamma X$. By the definition of support point, there exists $g \in A(X, E)$ such that $\operatorname{cl}_{\gamma X} c(g) \subset U$ and $(T g)\left(y_{0}\right) \neq 0$. Thus $c(T g)$ is an open neighborhood of $y_{0}$. By Corollary 4.5, $h(c(T g)) \subset U$ and we are done.

On the other hand, taking into account that $T$ is injective, a similar reasoning yields that for every open subset $U$ of $\gamma X$, there are points $h(y)$ in $U$, and consequently the range of $h$ is dense in $\gamma X$.

Lemma 4.7. The map $h$ can be extended to a continuous map from $\gamma Y$ onto $\gamma X$.

Proof. Obviously, as a consequence of the previous lemma, we can extend $h$ to a continuous map $\widehat{h}$ from $\beta Y$ onto $\gamma X$, so the result is true if $\gamma Y=\beta Y$.

Therefore we assume that $\gamma Y \neq \beta Y$.

Claim 1. Let $y_{0} \in \beta Y$. Given an open neighborhood $U$ of $\widehat{h}\left(y_{0}\right)$ in $\gamma X$, there exists $f \in A(X, E)$ such that $c(f) \subset U$ and $\|T f\|^{\beta Y}\left(y_{0}\right) \neq 0$.

Notice that, by hypothesis, since $\gamma Y \neq \beta Y$, there exists $g \in A(Y, F)$ such that $\|g\|^{\beta Y}\left(y_{0}\right) \neq 0$. Now take $k \in A$ such that $k^{\gamma Y} \equiv 0$ outside $U$ and $k^{\gamma Y} \equiv 1$ on an open neighborhood, say $V$, of $\widehat{h}\left(y_{0}\right)$. It is clear that, if we define $f:=k T^{-1} g$, then $c(f) \subset U$. Also, $\widehat{h}^{-1}(V)$ is a neighborhood of $y_{0}$. Now, if we take $y \in \widehat{h}^{-1}(V) \cap Y$, then $h(y) \in V$, and consequently $\left\|f-T^{-1} g\right\|^{\gamma X} \equiv 0$ on a neighborhood of $h(y)$. By Lemma 4.4, $(T f)(y)=g(y)$. Since this holds for every $y \in \widehat{h}^{-1}(V) \cap Y$, we conclude that $\|T f\|^{\beta Y}\left(y_{0}\right)=\|g\|^{\beta Y}\left(y_{0}\right) \neq 0$.

Claim 2. Given $y_{1}, y_{2} \in \beta Y$, if $y_{1} \sim y_{2}$, then $\widehat{h}\left(y_{1}\right)=\widehat{h}\left(y_{2}\right)$.

Suppose that $y_{1} \sim y_{2}, y_{1} \neq y_{2}$, and $\widehat{h}\left(y_{1}\right) \neq \widehat{h}\left(y_{2}\right)$. Take disjoint open subsets $U$ and $V$ of $\widehat{h}\left(y_{1}\right)$ and $\widehat{h}\left(y_{2}\right)$, respectively, in $\gamma X$. Now, by Claim 1, there exist $f_{1}, f_{2} \in A(X, E)$ such that $c\left(f_{1}\right) \subset U, c\left(f_{2}\right) \subset V$, and $\left\|T f_{1}\right\|^{\beta Y}\left(y_{1}\right) \neq 0,\left\|T f_{2}\right\|^{\beta Y}\left(y_{2}\right) \neq 0$. But, since $T$ is separating and $c\left(f_{1}\right) \cap c\left(f_{2}\right)=\emptyset$, we deduce that $\left\|\left(T f_{1}\right)(y)\right\| \cdot\left\|\left(T f_{2}\right)(y)\right\|=0$ for every $y \in Y$. Clearly this forces $\left\|T f_{1}\right\|^{\beta Y}\left(y_{2}\right)=0$. This contradicts the fact that $A(Y, F)$ is compatible with $B$, which means in particular that $\|g\|^{\beta Y}\left(y_{1}\right)=\|g\|^{\beta Y}\left(y_{2}\right)$ for every $g \in A(Y, F)$. 
Finally, because of Claim 2, given $y \in \gamma Y$ we can define the image of $y$ as the image under $\widehat{h}$ of any of the elements of its equivalence class. It is clear that this determines a surjective continuous map from $\gamma Y$ onto $\gamma X$ which is an extension of $h$.

The extension map given in Lemma 4.7 will also be called $h$.

Proof of Theorem 3.1. We will show that $h$ is a homeomorphism. We are going to find an inverse for $h$. It is clear that since $T$ is biseparating, we can construct a function $k: \gamma X \rightarrow \gamma Y$ associated to $T^{-1}$, which is an extension of a map from $X$ into $\gamma Y$ sending each point of $X$ to its support point for $T^{-1}$. We just have to prove that $k$ is the inverse of $h$.

Claim 1. If $y_{0} \in Y$, then $k\left(h\left(y_{0}\right)\right)=y_{0}$.

Assuming the contrary, suppose that $y_{0} \in Y$ satisfies $y_{0} \neq k\left(h\left(y_{0}\right)\right)$. Then consider two open neighborhoods $U, V$ in $\gamma Y$ of $y_{0}$ and $k\left(h\left(y_{0}\right)\right)$ respectively such that $U \cap V=\emptyset$.

Next, applying Lemma 4.1, take $f \in A(Y, F)$ such that $c(f) \subset U$ and $f\left(y_{0}\right) \neq 0$. By Corollary $4.5, h\left(y_{0}\right)$ belongs to $\mathrm{cl}_{\gamma X} c\left(T^{-1} f\right)$.

On the other hand, as $k: \gamma X \rightarrow \gamma Y$ is continuous there exists an open neighborhood $U_{1}$ of $h\left(y_{0}\right)$ in $\gamma X$ such that if $x \in U_{1}$, then $k(x) \in V$. Also since $h\left(y_{0}\right) \in \operatorname{cl}_{\gamma X} c\left(T^{-1} f\right)$, we have $U_{1} \cap c\left(T^{-1} f\right) \neq \emptyset$, so we may take

$$
x_{0} \in U_{1} \cap c\left(T^{-1} f\right) \text {. }
$$

Since $x_{0} \in U_{1}, k\left(x_{0}\right) \in V$. But $x_{0}$ also belongs to $X$, and therefore, by the definition of support point, there exists $g \in A(Y, F)$ such that $c(g) \subset V$ and $\left(T^{-1} g\right)\left(x_{0}\right) \neq 0$.

So we have $c(f) \cap c(g)=\emptyset$. But it is clear that

$$
x_{0} \in c\left(T^{-1} f\right) \cap c\left(T^{-1} g\right),
$$

contradicting the fact that $T^{-1}$ is separating.

Thus Claim 1 is proved.

Claim 2. If $y_{0} \in \gamma Y-Y$, then $k\left(h\left(y_{0}\right)\right)=y_{0}$.

Take a net $\left(y_{\alpha}\right)$ in $Y$ converging to $y_{0} \in \gamma Y-Y$. Since both $h$ and $k$ are continuous, the net $\left(k\left(h\left(y_{\alpha}\right)\right)\right)$ converges to $k\left(h\left(y_{0}\right)\right)$. But by Claim 1 , $k\left(h\left(y_{\alpha}\right)\right)=y_{\alpha}$ for every $\alpha$. This implies that this net converges to $y_{0}$, and consequently $k\left(h\left(y_{0}\right)\right)=y_{0}$. So Claim 2 is proved.

As a consequence we easily conclude that $k$ is the inverse map of $h$, and that both are homeomorphisms.

Proof of Corollary 3.2. By the previous theorem, $\beta X$ and $\beta Y$ are homeomorphic. Also, since the only points of $\beta X$ having a countable base of neighborhoods belong to $X([5,9.7])$, and the same applies to $Y$, we easily conclude that $X$ and $Y$ are homeomorphic. Finally, in the special case when 
$X$ and $Y$ are open subsets of $\mathbb{R}^{p}$ and $\mathbb{R}^{q}$, respectively, this fact implies that $p=q($ see for instance $[4$, p. 120]).

Proof of Corollary 3.3. In [2, Lemma 3.4], it is proved, in a different context, that every point of $X$ is a $G_{\delta^{-}}$set in $\gamma X$, and that, on the other hand, no point in $\gamma X-X$ is a $G_{\delta}$-set in $\gamma X$. Consequently, $h$ is a homeomorphism from $Y$ onto $X$.

Thus we have a map $S: C(\gamma X) \rightarrow C(\gamma Y)$ defined as $(S f)(y)=f(h(y))$ for every $f \in C(\gamma X)$ and every $y \in \gamma Y$. This map is easily seen to be bijective. Since $C_{\mathrm{u}}^{*}(X)$ and $C_{\mathrm{u}}^{*}(Y)$ can be identified with $C(\gamma X)$ and $C(\gamma Y)$, respectively (see Example 2), we may consider $S$ as a map from $C_{\mathrm{u}}^{*}(X)$ onto $C_{\mathrm{u}}^{*}(Y)$, where it is also defined as $S f=f \circ h$. As a consequence, for each $f \in C_{\mathrm{u}}^{*}(X), f \circ h$ belongs to $C_{\mathrm{u}}^{*}(Y)$.

But, on the other hand, we can prove as in [7, Theorem 2.3] (see also the Remark after it) that if $f \circ h \in C_{\mathrm{u}}^{*}(Y)$ whenever $f \in C_{\mathrm{u}}^{*}(X)$, then $h$ is uniformly continuous. Since the same argument works also for $h^{-1}$, the corollary is proved.

5. Proofs II. In this section we will assume that we are in Situations 2 or 3 .

Recall that in this context, if we look at the equivalence relation introduced in Section 2, then $\gamma X=\beta X$ and $\gamma Y=\beta Y$, so the previous results can be applied.

We start with a result whose proof is easy from Lemma 4.4 and the fact that $h$ is a homeomorphism.

Lemma 5.1. Let $T: A(X, E) \rightarrow A(Y, F)$ be a biseparating map. Suppose that $y \in \beta Y$, and that $f \in A(X, E)$ satisfies $\|f\|^{\beta X} \equiv 0$ on an open subset $U$ of $\beta X$. Then $\|T f\|^{\beta Y} \equiv 0$ on the open set $h^{-1}(U) \subset \beta Y$.

Proposition 5.2. If $T: A(X, E) \rightarrow A(Y, F)$ is a biseparating map, then for every $y \in Y, h(y) \in v X$.

Proof. Take $y_{0} \in Y$, and suppose that $h\left(y_{0}\right) \in \beta X-v X$. Then there exists a sequence $\left(U_{n}\right)$ of open neighborhoods of $h\left(y_{0}\right)$ in $\beta X$ such that $\operatorname{cl}_{\beta X} U_{n+1} \subset U_{n}, \operatorname{cl}_{\beta X} U_{n+1} \neq U_{n}$ for every $n \in \mathbb{N}$ and $X \cap \bigcap_{n=1}^{\infty} U_{n}=\emptyset$ (see for instance [3, Theorem 3.11.10]). It is clear that

$$
h\left(y_{0}\right) \in \operatorname{cl}_{\beta X} \bigcup_{n \in \mathbb{N}}\left(U_{n}-\operatorname{cl}_{\beta X} U_{n+2}\right) .
$$

Since $h$ is a homeomorphism, we see that $y_{0} \in \operatorname{cl}_{\beta Y} \bigcup_{n \in \mathbb{N}} h^{-1}\left(U_{n}-\operatorname{cl}_{\beta X} U_{n+2}\right)$. Consequently, if we define

$$
V_{1}:=\bigcup_{n \in \mathbb{N}} h^{-1}\left(U_{4 n}-\operatorname{cl}_{\beta X} U_{4 n+2}\right),
$$




$$
\begin{aligned}
V_{2} & :=\bigcup_{n \in \mathbb{N}} h^{-1}\left(U_{4 n-1}-\operatorname{cl}_{\beta X} U_{4 n+1}\right), \\
V_{3} & :=\bigcup_{n \in \mathbb{N}} h^{-1}\left(U_{4 n-2}-\operatorname{cl}_{\beta X} U_{4 n}\right), \\
V_{4} & :=\bigcup_{n \in \mathbb{N}} h^{-1}\left(U_{4 n-3}-\operatorname{cl}_{\beta X} U_{4 n-1}\right),
\end{aligned}
$$

then $y_{0}$ belongs to one of $\operatorname{cl}_{\beta Y} V_{1}, \mathrm{cl}_{\beta Y} V_{2}, \mathrm{cl}_{\beta Y} V_{3}$ or $\mathrm{cl}_{\beta Y} V_{4}$. We assume without loss of generality that $y_{0} \in \operatorname{cl}_{\beta Y} V_{1}$.

At this point we split the proof into two cases.

- Case 1. Assume we are in Situation 3.

It is clear that if we define $W_{n}:=h^{-1}\left(U_{4 n}-\operatorname{cl}_{\beta X} U_{4 n+2}\right)$ for each $n \in \mathbb{N}$, then $\mathrm{cl} W_{n} \cap \operatorname{cl} W_{m}=\emptyset$ for $n \neq m$. Consider now a sequence $\left(g_{n}\right)$ in $B$ such that, for every $n \in \mathbb{N}, 0 \leq g_{n} \leq 1, g_{n} \equiv 1$ on $Y \cap W_{n}$, and $g_{n} \equiv 0$ outside $Y \cap h^{-1}\left(U_{4 n-1}-\mathrm{cl}_{\beta X} U_{4 n+3}\right)$.

Applying Riesz's Lemma ([8, Theorem 1.3.2]), we can take a sequence $\left(\mathbf{e}_{n}\right)$ of norm one points in $F$ such that $\left\|\mathbf{e}_{n}-\mathbf{e}_{m}\right\| \geq 1 / 2$ for $n \neq m$.

It is also clear that, since $T^{-1}$ is separating, we have

$$
c\left(T^{-1}\left(g_{n} \mathbf{e}_{n}\right)\right) \cap c\left(T^{-1}\left(g_{m} \mathbf{e}_{m}\right)\right)=\emptyset
$$

if $n \neq m$. Define $f:=\sum_{n=1}^{\infty} T^{-1}\left(g_{n} \mathbf{e}_{n}\right)$. Notice that, for each $x \in X, f(x)$ belongs to $E$.

Claim 1. $f$ is continuous.

Notice that $\left\|g_{n} \mathbf{e}_{n}\right\|^{\beta Y} \equiv 0$ outside $\operatorname{cl}_{\beta Y} h^{-1}\left(U_{4 n-1}-\operatorname{cl}_{\beta X} U_{4 n+3}\right)$ for each $n$, which implies by Lemma 5.1 applied to $T^{-1}$ that

$$
\left\|T^{-1}\left(g_{n} \mathbf{e}_{n}\right)\right\|^{\beta X} \equiv 0
$$

outside $\operatorname{cl}_{\beta X}\left(U_{4 n-1}-\operatorname{cl}_{\beta X} U_{4 n+3}\right)$. That is, for every $n \in \mathbb{N}$, we have $c\left(T^{-1}\left(g_{n} \mathbf{e}_{n}\right)\right) \subset \operatorname{cl}_{\beta X}\left(U_{4 n-1}-\operatorname{cl}_{\beta X} U_{4 n+3}\right)$. Now given any $x \in X$, there exists an open neighborhood $U$ of $x$ in $X$ such that there are just a few numbers $k \in \mathbb{N}$ satisfying $U \cap \operatorname{cl}_{\beta X}\left(U_{4 k-1}-\operatorname{cl}_{\beta X} U_{4 k+3}\right) \neq \emptyset$, due to the construction of the sequence $\left(U_{n}\right)$. This proves Claim 1.

Claim 2. $f$ belongs to $A(X, E)$.

To prove this, we just need to show that $f$ is bounded. Suppose on the contrary that the sequence $\left(a_{n}:=\left\|T^{-1}\left(g_{n} \mathbf{e}_{n}\right)\right\|\right)$ is not bounded. Passing to a subsequence, we can assume that $a_{n} \geq n^{3}$ for every $n \in \mathbb{N}$. Next consider the map

$$
g:=\sum_{n \in \mathbb{N}} \frac{g_{n} \mathbf{e}_{n}}{n^{2}} .
$$


It is clear that, since each $\left\|g_{n} \mathbf{e}_{n}\right\| \leq 1$, the function $g$ belongs to $A(Y, F)$. Consequently, $T^{-1} g$ exists and is a bounded function on $X$. We are going to see that this leads to a contradiction. To this end take, for each $n \in \mathbb{N}$, $x_{n} \in X$ such that

$$
\left\|\left(T^{-1}\left(g_{n} \mathbf{e}_{n}\right)\right)\left(x_{n}\right)\right\| \geq a_{n} / 2 \geq n^{3} / 2 .
$$

Now notice that, for each $n_{0} \in \mathbb{N}$,

$$
g=\frac{g_{n_{0}} \mathbf{e}_{n_{0}}}{n_{0}^{2}}+\sum_{n \neq n_{0}} \frac{g_{n} \mathbf{e}_{n}}{n^{2}},
$$

where

$$
c\left(\frac{g_{n_{0}} \mathbf{e}_{n_{0}}}{n_{0}^{2}}\right) \cap c\left(\sum_{n \neq n_{0}} \frac{g_{n} \mathbf{e}_{n}}{n^{2}}\right)=\emptyset .
$$

Since $T^{-1}$ is separating, we deduce that

$$
c\left(T^{-1}\left(\frac{g_{n_{0}} \mathbf{e}_{n_{0}}}{n_{0}^{2}}\right)\right) \cap c\left(T^{-1} \sum_{n \neq n_{0}} \frac{g_{n} \mathbf{e}_{n}}{n^{2}}\right)=\emptyset,
$$

and this implies that

$$
\left\|\left(T^{-1} g\right)\left(x_{n_{0}}\right)\right\|=\frac{1}{n_{0}^{2}}\left\|\left(T^{-1}\left(g_{n_{0}} \mathbf{e}_{n_{0}}\right)\right)\left(x_{n_{0}}\right)\right\| \geq \frac{n_{0}}{2} .
$$

Since this applies to every $n_{0} \in \mathbb{N}$, we deduce that $T^{-1} g$ is not bounded. This contradiction shows that $f$ is bounded, and Claim 2 is proved.

Consequently, $T f$ belongs to $A(Y, F)$, and for each $n \in \mathbb{N}$,

$$
\left\|f-T^{-1}\left(g_{n} \mathbf{e}_{n}\right)\right\|^{\beta X} \equiv 0
$$

on $U_{4 n}-\mathrm{cl}_{\beta X} U_{4 n+2}$, which implies by Lemma 5.1 that

$$
T f \equiv g_{n} \mathbf{e}_{n} \equiv \mathbf{e}_{n}
$$

in $Y \cap W_{n}$. Also, suppose that $(T f)\left(y_{0}\right)=\mathbf{e}_{0} \in F$. Then there exists a neighborhood $U$ of $y_{0}$ in $Y$ such that if $y, y^{\prime} \in U$, then

$$
\left\|(T f)(y)-(T f)\left(y^{\prime}\right)\right\|<1 / 4 .
$$

On the other hand, recall that $y_{0} \in \operatorname{cl}_{\beta Y} V_{1}$ and, since $h\left(y_{0}\right) \notin \operatorname{cl}_{\beta X}\left(U_{n}-\right.$ $\left.\operatorname{cl}_{\beta X} U_{n+2}\right)$ for any $n \in \mathbb{N}$, it follows that $y_{0} \notin \operatorname{cl}_{\beta Y} W_{n}$ for any $n \in \mathbb{N}$. This implies that for every $k \in \mathbb{N}$,

$$
y_{0} \in \operatorname{cl}_{\beta Y} \bigcup_{n \geq k} W_{n} .
$$

As a consequence, there are $n_{1}, n_{2} \in \mathbb{N}, n_{1} \neq n_{2}$, such that $U \cap W_{n_{1}} \neq \emptyset \neq$ $U \cap W_{n_{2}}$, and hence there are $y_{n_{1}} \in U \cap W_{n_{1}}, y_{n_{2}} \in U \cap W_{n_{2}}$. Then

$$
1 / 4>\left\|(T f)\left(y_{n_{1}}\right)-(T f)\left(y_{n_{2}}\right)\right\|=\left\|\mathbf{e}_{n_{1}}-\mathbf{e}_{n_{2}}\right\| \geq 1 / 2,
$$

which is impossible. 
- Case 2. Assume we are in Situation 2.

We follow a similar pattern of proof. Now consider a sequence $\left(f_{n}\right)$ in $A$ such that, for every $n \in \mathbb{N}, c\left(f_{n}\right) \subset X \cap U_{4 n-2}$, and $f_{n}(x)=1$ for every $x \in X \cap U_{4 n}$. Define $g:=\sum_{n=1}^{\infty} f_{n}$. Since $A$ is local, it is easy to check that $g$ belongs to $A$. Also it is easy to see that for every $n \in \mathbb{N}, g$ is constantly equal to $n$ on $X \cap\left(U_{4 n}-\mathrm{cl}_{\beta X} U_{4 n+2}\right)$. Next take $f \in A(X, E)$ such that $(T f)\left(y_{0}\right)=\mathbf{f}_{0} \neq 0$. Suppose that $T(g f)\left(y_{0}\right)=\mathbf{f}_{1} \in F$. Consider $n_{0} \in \mathbb{N}$, $n_{0}\left\|\mathbf{f}_{0}\right\| / 2>\left\|\mathbf{f}_{1}\right\|+1$, and an open neighborhood $U\left(y_{0}\right)$ of $y_{0}$ in $\beta Y$ such that $h\left(U\left(y_{0}\right)\right) \subset U_{4 n_{0}} \cap V$, where

$$
V:=\left\{x \in \beta X:\|T f\|^{\beta Y}\left(h^{-1}(x)\right)>\left\|\mathbf{f}_{0}\right\| / 2\right\} .
$$

Since $h\left(y_{0}\right)$ belongs to $\operatorname{cl}_{\beta X} V_{1}$, we deduce as above that for every $k \in \mathbb{N}$,

$$
y_{0} \in \operatorname{cl}_{\beta Y} \bigcup_{n \geq k} W_{n} .
$$

Now it is not difficult to see that there exists $k \in \mathbb{N}, k \geq n_{0}$, such that $h\left(U\left(y_{0}\right)\right) \cap\left(U_{4 k}-\operatorname{cl}_{\beta X} U_{4 k+2}\right)$ is nonempty. Then if for some $y_{1} \in U\left(y_{0}\right) \cap Y$, $h\left(y_{1}\right)$ belongs to $U_{4 k}-\mathrm{cl}_{\beta X} U_{4 k+2}$, we infer that $\|g f-k f\|^{\beta X}$ is constantly zero in a neighborhood of $h\left(y_{1}\right)$. This means, in view of Lemma 5.1, that $T(g f-k f)\left(y_{1}\right)=0$, and consequently,

$$
\left\|T(g f)\left(y_{1}\right)\right\|=k\left\|(T f)\left(y_{1}\right)\right\| \geq n_{0}\left\|\mathbf{f}_{0}\right\| / 2 \geq\left\|\mathbf{f}_{1}\right\|+1 .
$$

Since this happens for every open neighborhood of $y_{0}$, we deduce that $T(g f)$ is not continuous, which is not possible.

In both cases, we conclude that $h(y)$ belongs to $v X$ for every $y \in Y$.

Proof of Theorem 3.4. The restriction of $h$ to $v Y$ is continuous. Also, by Proposition 5.2, $h(y)$ belongs to $v X$ for every $y \in Y$. Since $v X$ is realcompact, we deduce that $h(v Y)$ is contained in $v X$. Since $h^{-1}$, for the same reason, maps elements of $v X$ to elements of $v Y$, and $h^{-1}$ is continuous, we conclude that the restriction of $h$ to $v Y$ is a homeomorphism onto $v X$.

The author wishes to thank the referee and Professor K. Jarosz for some suggestions which improved this paper.

\section{References}

[1] J. Araujo, E. Beckenstein and L. Narici, Biseparating maps and homeomorphic realcompactifications, J. Math. Anal. Appl. 192 (1995), 258-265.

[2] J. Araujo and J. J. Font, Linear isometries on subalgebras of uniformly continuous functions, Proc. Edinburgh Math. Soc. 43 (2000), 139-147.

[3] R. Engelking, General Topology, Heldermann, Berlin, 1989.

[4] V. V. Fedorchuk, The fundamentals of dimension theory, in: General Topology I, A. V. Arkhangel'skiı̌ and L. S. Pontryagin (eds.), Springer, Berlin, 1990, 91-195. 
[5] L. Gillman and M. Jerison, Rings of Continuous Functions, Van Nostrand, Princeton, 1960 .

[6] M. Henriksen, On the equivalence of the ring, lattice and semigroup of continuous functions, Proc. Amer. Math. Soc. 7 (1956), 959-960.

[7] M. Lacruz and J. G. Llavona, Composition operators between algebras of uniformly continuous functions, Arch. Math. (Basel) 69 (1997), 52-56.

[8] R. Larsen, Functional Analysis. An introduction, Dekker, New York, 1973.

[9] J. Wloka, Partial Differential Equations, Cambridge Univ. Press, Cambridge, 1987.

Departamento de Matemáticas, Estadística y Computación

Facultad de Ciencias

Universidad de Cantabria

Avenida de los Castros s.n.

E-39071 Santander, Spain

E-mail: araujoj@unican.es

Received 2 August 2000;

in revised form 18 June 2001 